\title{
Approximation properties related to the Bell polynomials
}

\author{
IOAN GAVREA AND MIRCEA IVAN*
}

ABSTRACT. The authors provide a complete asymptotic expansion for a class of functions in terms of the complete Bell polynomials. In particular, they obtain known asymptotic expansions of some Keller type sequences.

Keywords: Asymptotic expansions, Bell polynomials.

2020 Mathematics Subject Classification: 41A60.

Dedicated to Professor Francesco Altomare, on occasion of his 70th birthday, with esteem and friendship.

\section{INTRODUCTION}

The first references to the number $e$ were published in 1618 in the table of an appendix of a work on logarithms by John Napier [1, p. xiii]. The discovery of the constant itself is credited to Jacob Bernoulli in 1690 who considered the problem of continuous compounding of interest,

$$
e=\lim _{n \rightarrow \infty}\left(1+\frac{1}{n}\right)^{n}
$$

Leonhard Euler introduced the letter $e$ as the base for natural logarithms, writing in a letter to Christian Goldbach on 25 November 1731. In 1665, Newton [1, p. 151] discovered

$$
e=1+\frac{1}{1 !}+\frac{1}{2 !}+\frac{1}{3 !}+\cdots
$$

Let us consider the sequence

$$
(n+1)\left(1+\frac{1}{n+1}\right)^{n+1}-n\left(1+\frac{1}{n}\right)^{n}, \quad n=1,2, \ldots
$$

The sequence (1.1) is attributed to Felix A. Keller (see, e.g., [2], [3, p. 14], but its origin surely lay in the Euler age). In 1998, H. J. Brothers and J. A. Knox [4, Eq. (8)] gave the following approximation to $e$,

$$
\begin{aligned}
& \frac{(x+1)^{x+1}}{x^{x}}-\frac{x^{x}}{(x-1)^{x-1}} \\
= & (1+x)\left(1+\frac{1}{x}\right)^{x}+(1-x)\left(1-\frac{1}{x}\right)^{-x} \\
= & e\left(1+\frac{1}{24 x^{2}}+\frac{11}{640 x^{4}}+\frac{5525}{580608 x^{6}}+\mathcal{O}\left(\frac{1}{x^{8}}\right)\right), \quad x \rightarrow \infty .
\end{aligned}
$$

Received: 14.01.2021; Accepted: 27.02.2021; Published Online: 01.03.2021

*Corresponding author: Mircea Ivan; mircea.ivan@math.utcluj.ro

DOI: $10.33205 / \mathrm{cma} .861342$ 
We recall an excellent result of Alzer and Berg [5, (2.2)]:

$$
(x+1)\left(e-\left(1+\frac{1}{x}\right)^{x}\right)=\frac{e}{2}+\frac{1}{\pi} \int_{0}^{1} \frac{s^{s}(1-s)^{1-s} \sin (\pi s)}{x+s} \mathrm{~d} s, \quad x>0 .
$$

Next, using the identity

$$
\frac{1}{x+s}=\frac{1}{x+a} \sum_{n=0}^{\infty}\left(\frac{a-s}{x+a}\right)^{n}, \quad a \geq 0,|a-s|<|x+a|, \text { for } s \in[0,1],
$$

from (1.2), we deduce that

$$
\begin{aligned}
& (x+1)\left(e-\left(1+\frac{1}{x}\right)^{x}\right) \\
= & \frac{e}{2}+\frac{1}{\pi} \sum_{n=0}^{\infty} \frac{1}{(x+a)^{n+1}} \int_{0}^{1} s^{s}(1-s)^{1-s} \sin (\pi s)(a-s)^{n} \mathrm{~d} s .
\end{aligned}
$$

For $a=\frac{11}{12},(1.3)$ yields the result of Mortici and $\mathrm{Hu}[6,(3.1)]$;

for $a=1,(1.3)$ gives an expansion in [7];

for $a=0, x>1,(1.3)$ becomes

$$
(x+1)\left(e-\left(1+\frac{1}{x}\right)^{x}\right)=\frac{e}{2}+\frac{1}{\pi} \sum_{n=0}^{\infty} \frac{(-1)^{n}}{x^{n+1}} \int_{0}^{1} s^{s}(1-s)^{n+1-s} \sin (\pi s) \mathrm{d} s .
$$

We will review the integrals involved in (1.4) in subsection 3.1. The main result of the paper is the series expansion (2.9) of the function $x \mapsto\left(1+\frac{1}{x+a}\right)^{x+b}$ in terms of Bell polynomials. This extends many known results.

1.1. Complete asymptotic expansion. Let $\left(b_{n}\right)_{n \geq 0}$ be a sequence of real numbers and $f:(0, \infty) \rightarrow$ $\mathbb{R}$. Use the symbol $\mathcal{O}$ for Landau's big " $\mathrm{O}$ " notation. We recall that $\sum_{n=0}^{\infty} \frac{b_{n}}{x^{n}}$ is said to be a complete asymptotic expansion of $f$ as $x \rightarrow \infty$, and use the notation

$$
f(x) \sim \sum_{n=0}^{\infty} \frac{b_{n}}{x^{n}}, \quad \text { as } x \rightarrow \infty,
$$

if

$$
f(x)=\sum_{n=0}^{p} \frac{b_{n}}{x^{n}}+\mathcal{O}\left(x^{-p-1}\right), \quad \text { as } x \rightarrow \infty
$$

for all integers $p \geq 0$.

1.2. The Bell polynomials. Let $\left(x_{n}\right)_{n \geq 1}$ be a sequence of numbers. The complete exponential Bell polynomials $B_{n}\left(x_{1}, \ldots, x_{n}\right)$ (see, e.g., [8, Chapter 2, Section 8], [9, p. 134]) denoted in the sequel by $\operatorname{Bel}_{n}\left[x_{i}\right]$, are given by the formal series identity

$$
\exp \left(\sum_{i=1}^{\infty} x_{i} \frac{t^{i}}{i !}\right)=\sum_{n=0}^{\infty} \mathbf{B e l}_{n}\left[x_{i}\right] \frac{t^{n}}{n !} .
$$

They may be recursively defined as

$$
\operatorname{Bell}_{0}\left[x_{i}\right]:=1, \quad \operatorname{Bell}_{n+1}\left[x_{i}\right]=\sum_{j=0}^{n}\left(\begin{array}{c}
n \\
j
\end{array}\right) \mathbf{B e l}_{n-j}\left[x_{i}\right] x_{j+1}, \quad n=0,1, \ldots
$$


The following table can be obtained immediately from (1.6).

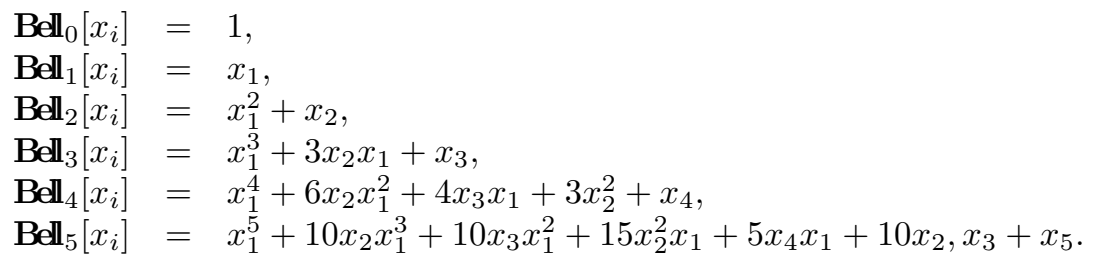

\section{MAIN Results}

It is well known that if $z \mapsto g(z)$ is holomorphic in the disk $|z|<R$, then $z \mapsto \exp (g(z))$ is holomorphic in the disk $|z|<R$. In consequence, the power series expansion of $\exp (g(z))$ has a radius of convergence at least $R$. So, if the power series $\sum_{i=1}^{\infty} x_{i} \frac{t^{i}}{i !}$ has the radius of convergence $R>0$, then the formal equality (1.5) becomes an equality

$$
\exp \left(\sum_{i=1}^{\infty} x_{i} \frac{t^{i}}{i !}\right)=\sum_{n=0}^{\infty} \operatorname{Bel}_{n}\left[x_{i}\right] \frac{t^{n}}{n !}, \quad|t|<R .
$$

The following is the main result of the paper.

Theorem 2.1. Let $a, b \in \mathbb{R}$. For

$$
x_{i, a, b}:=(-1)^{i} i !\left(\frac{(a+1)^{i+1}-a^{i+1}}{i+1}-\frac{b\left((a+1)^{i}-a^{i}\right)}{i}\right), \quad i=1,2, \ldots,
$$

we obtain the following equality

$$
\left(1+\frac{1}{x+a}\right)^{x+b}=e \sum_{n=0}^{\infty} \frac{\operatorname{Bel}_{n}\left[x_{i, a, b}\right]}{n !} \frac{1}{x^{n}}, \quad|x|>\max (|a|,|a+1|) .
$$

Proof. The following expansion can be obtained by simple calculation,

$$
\begin{aligned}
& (x+b) \log \left(1+\frac{1}{x+a}\right) \\
= & (x+b) \log \frac{1+\frac{a+1}{x}}{1+\frac{a}{x}} \\
= & 1+\sum_{i=1}^{\infty}(-1)^{i} i !\left(\frac{(a+1)^{i+1}-a^{i+1}}{i+1}-\frac{b\left((a+1)^{i}-a^{i}\right)}{i}\right) \frac{1}{i ! x^{i}},
\end{aligned}
$$

$|x|>\max (|a|,|a+1|)$. Using (2.7), the proof is complete.

In particular, we obtain: 
Example 2.1. The following asymptotic expansions hold true:

$$
\begin{aligned}
& \left(1+\frac{1}{x+a}\right)^{x+b} \\
= & e \\
- & \frac{e(2 a-2 b+1)}{2} \frac{1}{x} \\
+ & \frac{e\left(36 a^{2}-48 a b+36 a+12 b^{2}-24 b+11\right)}{24} \frac{1}{x^{2}} \\
- & \frac{e\left(104 a^{3}-168 a^{2} b+156 a^{2}+72 a b^{2}-168 a b+94 a-8 b^{3}+36 b^{2}-50 b+21\right)}{48} \frac{1}{x^{3}} \\
+ & \mathcal{O}\left(x^{-4}\right), \quad x \rightarrow \infty, \\
\qquad & \left(1+\frac{1}{x+a-\frac{1}{4}}\right)^{x+a+\frac{1}{4}}=e+\mathcal{O}\left(x^{-2}\right), \quad x \rightarrow \infty .
\end{aligned}
$$

3. Applications

All known or new results in this section stem from Theorem 2.1.

Corollary 3.1. From (2.9), we deduce

$$
(x+c)\left(1+\frac{1}{x+a}\right)^{x+b}-e x=e \sum_{k=0}^{\infty}\left(\frac{\mathbf{B e l}_{k+1}\left[x_{i, a, b}\right]}{(k+1) !}+c \frac{\operatorname{Bel}_{k}\left[x_{i, a, b}\right]}{k !}\right) \cdot \frac{1}{x^{k}}
$$

and, in particular,

$$
\begin{aligned}
& (x+c)\left(\frac{1}{x+a}+1\right)^{x+b}-e x \\
= & -\frac{1}{2} e(2 a-2 b-2 c+1) \\
+ & e\left(36 a^{2}-48 a b-24 a c+36 a+12 b^{2}+24 b c-24 b-12 c+11\right) \frac{1}{24 x} \\
- & e\left(104 a^{3}-168 a^{2} b-72 a^{2} c+156 a^{2}+72 a b^{2}+96 a b c-168 a b\right. \\
- & \left.72 a c+94 a-8 b^{3}-24 b^{2} c+36 b^{2}+48 b c-50 b-22 c+21\right) \frac{1}{48 x^{2}} \\
+ & \mathcal{O}\left(x^{-3}\right) .
\end{aligned}
$$

We note that particular cases of (2.9) can be found, e.g., in papers of H. J. Brothers and J. A. Knox [4, 10], C. Mortici and X.-J. Jang [11], C. Mortici and Y. Hu [6].

3.1. Evaluating the integrals in (1.4). In this subsection, we obtain the following evaluation of the integrals involved in (1.4).

\section{Proposition 3.1.}

$$
J_{k}:=\int_{0}^{1} s^{s}(1-s)^{k-s} \sin (\pi s) \mathrm{d} s=(-1)^{k} \pi e\left(\frac{\mathbf{B e l}_{k+1}\left[x_{i, 0,0}\right]}{(k+1) !}+\frac{\operatorname{Bel}_{k}\left[x_{i, 0,0}\right]}{k !}\right),
$$

$k=1,2, \ldots$. 
Proof. Taking $a=0, b=0$, and $c=1$ in (3.11), we obtain

$$
(x+1)\left(1+\frac{1}{x}\right)^{x}-e x=e \sum_{k=0}^{\infty}\left(\frac{\operatorname{Bel}_{k+1}\left[x_{i, 0,0}\right]}{(k+1) !}+\frac{\operatorname{Bel}_{k}\left[x_{i, 0,0}\right]}{k !}\right) \cdot \frac{1}{x^{k}}, \quad x>1,
$$

where

$$
x_{0,0, i}=\frac{(-1)^{i} i !}{i+1}, \quad i=1,2, \ldots
$$

Comparing (1.4) with (3.13), we succeeded in calculating the integrals (3.12).

For example,

$$
\begin{aligned}
\int_{0}^{1} s^{s}(1-s)^{1-s} \sin (\pi s) \mathrm{d} s=J_{1} & =\frac{e \pi}{24}, \\
J_{2} & =\frac{e \pi}{48}, \\
J_{3} & =\frac{73 e \pi}{5760}, \\
J_{4} & =\frac{11 e \pi}{1280} .
\end{aligned}
$$

Note that MATHEMATICA and other assistant software failed to evaluate the integrals (3.12).

3.2. A generalized Keller function. Extend now the Keller sequence (1.1) to the function

$$
K(a, b, c ; x):=(x+c)\left(1+\frac{1}{x+a}\right)^{x+b}-(x+c-1)\left(1+\frac{1}{x+a-1}\right)^{x+b-1}-e,
$$

$|x|>\max (|a-1|,|a|,|a+1|)=|a|+1$.

From (3.11), we obtain

$$
\begin{aligned}
K(a, b, c ; x)=e \sum_{k=2}^{\infty} \frac{1}{x^{k}}( & \frac{c \mathbf{B e l}_{k}\left[x_{i, a, b}\right]-(c-1) \mathbf{B e l}_{k}\left[x_{i, a-1, b-1}\right]}{k !} \\
& \left.+\frac{\mathbf{B e l}_{k+1}\left[x_{i, a, b}\right]-\mathbf{B e l}_{k+1}\left[x_{i, a-1, b-1}\right]}{(k+1) !}\right),
\end{aligned}
$$

$|x|>|a|+1$. We note that, for any parameters $a, b, c \in \mathbb{R}$, the function $K(a, b, c ; x)$ is a $\mathcal{O}\left(x^{-2}\right)$, as $x \rightarrow \infty$. For example,

$$
\begin{aligned}
& K(a, b, c ; x) \\
= & \frac{e}{24 x^{2}} \cdot\left(-36 a^{2}+48 a b+24 a c-36 a-12 b^{2}-24 b c+24 b+12 c-11\right) \\
+ & \frac{e}{24 x^{3}} \cdot\left(104 a^{3}-168 a^{2} b-72 a^{2} c+84 a^{2}+72 a b^{2}+96 a b c-72 a b\right. \\
- & \left.24 a c+22 a-8 b^{3}-24 b^{2} c+12 b^{2}-2 b+2 c-1\right) \\
+ & \mathcal{O}\left(x^{-4}\right), \quad x \rightarrow \infty
\end{aligned}
$$


In particular, we obtain

$$
\begin{aligned}
K(a, a, 1 ; x) & =\frac{e-12 e a}{24 x^{2}}+\frac{e(4 a(6 a-1)+1)}{24 x^{3}}+\mathcal{O}\left(x^{-4}\right), \quad x \rightarrow \infty, \\
K\left(\frac{1}{12}, \frac{1}{12}, 1 ; x\right) & =\frac{5 e}{144 x^{3}}+\mathcal{O}\left(x^{-4}\right), \quad x \rightarrow \infty,
\end{aligned}
$$

which are cases considered in [7] and [11]. Taking benefit of three free parameters $a, b, c$, we obtain

$$
K\left(-\frac{1}{2}, \sqrt{\frac{1}{2}+\frac{1}{\sqrt{6}}},-\frac{1}{6} \sqrt{9+\sqrt{6}} ; x\right)=\frac{(3+5 \sqrt{6}) e}{720 x^{4}}+\mathcal{O}\left(x^{-5}\right) .
$$

3.3. On an expansion of Yang. In [12, Theorem 1], X. Yang obtained the following expansion

$$
\left(1+\frac{1}{x}\right)^{x}=e\left(1-\sum_{k=1}^{\infty} \frac{b_{k}}{(1+x)^{k}}\right), \quad x>0
$$

where

$$
b_{1}=\frac{1}{2}, \quad b_{k+1}=\frac{1}{k+1}\left(\frac{1}{k+2}-\sum_{i=1}^{k} \frac{b_{i}}{k+2-i}\right), \quad k=1,2, \ldots
$$

We prove that Yang's formula (3.17) is a particular case of the general Bell-type expansion (2.7) for

Indeed, we have

$$
t=\frac{1}{1+x} \quad \text { and } \quad x_{i}=-\frac{(i-1) !}{i+1}, \quad i=1,2, \ldots
$$

$$
\begin{aligned}
\left(1+\frac{1}{x}\right)^{x} e^{-1} & =(1-t)^{1-\frac{1}{t}} e^{-1}=\exp \left(\frac{(t-1) \log (1-t)-t}{t}\right) \\
& =\exp \left(-\sum_{i=1}^{\infty} \frac{t^{i}}{i(i+1)}\right) \\
& =\exp \left(-\sum_{i=1}^{\infty} \frac{(i-1) !}{i+1} \cdot \frac{t^{i}}{i !}\right)=\sum_{k=0}^{\infty} \frac{\mathrm{Be}_{k}\left[x_{i}\right]}{k !} \cdot t^{k}
\end{aligned}
$$

hence

$$
\left(1+\frac{1}{x}\right)^{x}=e \sum_{k=0}^{\infty} \frac{\mathbf{B e l}_{k}\left[x_{i}\right]}{k !} \cdot \frac{1}{(1+x)^{k}}, \quad x>0 .
$$

Acknowledgments. We thank the anonymous reviewers whose comments and suggestions improved the manuscript.

\section{REFERENCES}

[1] E. Maor: $e$ : the story of a number, Princeton University Press, Princeton, NJ (2009).

[2] J. Sandor: On certain limits related to the number e, Libertas Math., 20 (2000) 155-159, dedicated to Emeritus Professor Corneliu Constantinescu on the occasion of his 70th birthday.

[3] S. R. Finch: Mathematical constants, Vol. 94 of Encyclopedia of Mathematics and its Applications, Cambridge University Press, Cambridge (2003).

[4] H. J. Brothers, J. A. Knox: New closed-form approximations to the logarithmic constant e, Math. Intelligencer, 20 (4) (1998), 25-29. 
[5] H. Alzer, C. Berg: Some classes of completely monotonic functions, Ann. Acad. Sci. Fenn. Math., 27 (2) (2002), 445-460.

[6] C. Mortici, Y. Hu: On an infinite series for $(1+1 / x)^{x}$ (Jun 2014). http://arxiv. org/abs/1406.7814v1

[7] Y. Hu, C. Mortici: On the Keller limit and generalization, J. Inequal. Appl., 2016 (2016), 97.

[8] J. Riordan: An introduction to combinatorial analysis, Wiley Publications in Mathematical Statistics, John Wiley \& Sons, Inc., New York; Chapman \& Hall, Ltd., London (1958).

[9] L. Comtet: Advanced combinatorics, enlarged Edition, D. Reidel Publishing Co., Dordrecht (1974).

[10] J. A. Knox, H. J. Brothers: Novel series-based approximations to e, College Math. J., 30 (4) (1999), $269-275$.

[11] C. Mortici, X.-J. Jang: Estimates of $(1+x)^{1 / x}$ involved in Carleman's inequality and Keller's limit, Filomat, 29 (7) (2015), 1535-1539.

[12] X. Yang: Approximations for constant e and their applications, J. Math. Anal. Appl., 262 (2) (2001), 651-659.

\section{IOAN GAVREA}

TECHNICAL UNIVERSITY OF CLUJ-NAPOCA

DEPARTMENT OF MATHEMATICS

STR. MemorandumUlui NR. 28, 400114 Cluj-NAPOCA, ROMANiA

E-mail address: ioan. gavrea@math.utcluj.ro

Mircea IVAN

TECHNICAL UNIVERSITY OF CLUJ-NAPOCA

DEPARTMENT OF MATHEMATICS

Str. Memorandumului NR. 28, 400114 Cluj-NAPOCA, Romania

ORCID: 0000-0001-6047-2470

E-mail address: mircea. ivan@math.utcluj.ro 